\title{
Ventricular Aneurysm Resection
}

National Cancer Institute

\section{Source}

National Cancer Institute. Ventricular Aneurysm Resection. NCI Thesaurus. Code C80451.

Cardiac surgical procedure performed for the removal of an aneurysm from the ventricular wall. 